\title{
Study of Cardiac Features in Adults with Down Syndrome
}

\author{
Claudia Condoluci ${ }^{1}$, Manuela Galli ${ }^{1,2}$, Veronica Cimolin ${ }^{2, *}$, Chiara Rigoldi $^{2}$ and \\ Giorgio Albertini ${ }^{1}$
}

\author{
${ }^{1}$ IRCCS “San Raffaele Pisana”, Tosinvest Sanità, Rome, Italy \\ ${ }^{2}$ Department of Electronics, Information and Bioengineering, Politecnico di Milano, Milano, Italy
}

\begin{abstract}
Down Syndrome (DS) has a significant impact on the development of many tissues, most notably in the heart and in the brain. According to the clinical need to better investigate these aspects, the main aim of this study was to make an overview on the cardiac features in adult individuals with DS.

The following data of 37 individuals with DS (range: 18-60 years) were collected and analysed: age, gender, height, weight, waist to height ratio (WHER), smoking history and cardiac parameters (heart rate and blood pressure), blood glucose level or glycaemia, cholesterol (total, high-density lipoprotein - HDL - and low-density lipoprotein - LDL cholesterol) and triglycerides. In addition, their gait pattern were quantified with 3D Gait Analysis.

BMI, WHER and cholesterol HDL results were above the upper limits of the recommended range in people with DS; on the contrary, blood pressure, heart rate, glycaemia, cholesterol-LDL and triglycerides were within the recommended range. Comparing males and females in the DS group, the females showed statistically different values for cholesteroltotal, and triglycerides values, with lower values in comparison to males. In motor performance, people with DS presented abnormal gait patterns. Some significant correlations were found: age with LDL, WHER with weight and BMI, systolic with diastolic blood pressure, total cholesterol with LDL and triglycerides.
\end{abstract}

Our results showed that adults with DS suffer from a high prevalence of physical disorders, including overweight and obesity, and abnormal cholesterol values, together with abnormal gait pattern.

Keywords: Down Syndrome, cardiac features, gait, intellectual disability, rehabilitation.

\section{INTRODUCTION}

Down syndrome (DS) is caused by trisomy of the human chromosome 21 (Hsa21). Approximately 0.45\% of human conceptions are trisomic for Hsa21 [1]. The incidence of trisomy is influenced by maternal age and differs among populations (between 1 in 319 and 1 in 1000 live births are trisomic for Hsa21) [2-4]. Trisomicfetuses are at an elevated risk of miscarriage, and people with DS have an increased risk of developing several medical conditions [5]. Recent advances in medical treatment and social inclusion have significantly increased the life expectancy of people with DS: in economically developed countries, the average life span of people who are trisomic for Hsa21 is now greater than 55 years [6].

Trisomy of Hsa21 is associated with a number of features, occurring in all individuals, including mild-tomoderate learning disability, craniofacial abnormalities, hypotonia and movement disorders in early infancy [7]. Although these phenotypes are always found in people with DS, the degree to which an individual is affected varies. Additionally, trisomy of Hsa21 is also associated with variant phenotypes that affect individuals with DS

*Address correspondence to this author at the Dipartimento di Elettronica, Informazione e Bioingegneria, Politecnico di Milano, P.zza Leonardo da Vinci 32, 20133 Milano, Italy; Tel: +39 02 23993359;

E-mail: veronica.cimolin@polimi.it in different percentages, including atrioventricular septal defects (AVSDs) in the heart, acute megakaryoblastic leukaemia (AMKL) and a decrease in the incidence of some solid tumours. In addition, there are a number of medical problems associated with the syndrome, including obesity, muscular hypotonia, ligament laxity and orthopaedic problems, which are responsible for postural and gait alterations widely documented by the literature [8-20]. We hypothesize that these motor problems may be connected to the high fat percentage, together with other features common in individuals with DS, such as muscle hypotonia and ligament laxity. A recent study quantified in fact the differences during gait between obese individuals with DS and non obese individuals with DS [21]. The results of this study demonstrated that obesity in individuals with DS has quite impact on the gait strategy, and the main differences between obese and non obese individuals with DS are present particular at distal level (ankle joint) [21]. Trisomy of Hsa21 has then a significant impact on the development of many tissues, most notably in the heart and in the brain. Focusing attention on heart, trisomy of Hsa21 is associated with a number of congenital heart defects, the most common being AVSD that occurs in $\sim 20 \%$ of the people with DS [22] In addition, people with DS have been reported to have a reduced incidence of hypertension [23, 24]. 
From the literature, the association between the presence of congenital heart defects in people with DS is well known, but less is described about acquired cardiac risk factors. Prasher [25] assessed 201 adults with DS to ascertain the prevalence of medical problems. As concerns cardiac parameters, they found a low heart rate and low blood pressure recordings. However, to our knowledge no other more recent researches were conducted on this topic.

According to these considerations and to the clinical need to better investigate the aspects related to cardiac risk factors, the main aim of this study was twofold: first, to provide an overview of the cardiac features in a group of adult individuals with DS, then to search for a possible correlation between cardiac parameters, presence of obesity and gait alterations.

In this way, we aim to improve the knowledge about this area; in this wake of evidence, appropriate and effective intervention could be tailored to the unveiled specific features.

\section{MATERIALS AND METHODS}

The studied group consisted of 37 individuals with DS (mean age: $36.1 \pm 7.3$ years) admitted to the IRCCS San Raffaele Pisana, TosinvestSanità, Roma (Italy) for a multidisciplinary rehabilitation program. Patients were included in the study after a complete medical evaluation according to the following exclusion criteria: congenital or acquired cardiac pathologies, respiratory, haematological, metabolic and infectious diseases, presence of motor disorders.

The study was in accordance with the Helsinki Declaration of 1975. All participants were volunteers and written informed consent was obtained by all of them. Data from each individual included age, gender, height, weight, waist to height ratio (WHER), smoking history and cardiac parameters. For cardiac parameters, heart rate and blood pressure, glycaemia, cholesterol (total, high-density lipoprotein - HDL - and low-density lipoprotein - LDL- cholesterol) and triglycerides were collected. These parameters are included in the annual screening performed in the Institute on adult patients with Down Syndrome.

In addition, gait performance was instrumentally evaluated with 3D gait analysis, using an optoelectronic system with passive markers (BTS, Italy) and 2 TV camera synchronized Video system (BTS, Italy) for video recording. The overall gait deviation of each participant was quantified as a gait profile score (GPS)
[26]. The GPS was selected, as it is the most compound and neutral score in respect of the contributing parameter [27]. The GPS represents the difference between the individual's data and the average from the reference dataset; the higher the GPS value is, the less physiological gait pattern is.

All parameters were computed for each participant and the mean and standard deviation values of all indexes were calculated the group with DS. As the parameters were normally distributed, we used the ttest independent by groups to compare males and females within DS and the Pearson coefficient (r) to examine the relationship between clinical, cardiac and gait parameters of the entire group with DS. Statistical significance was set at $p<0.05$.

\section{RESULTS}

All the participants were able to complete clinical, cardiac and movement analysis evaluations. None smoked and none had had previous cardiac symptoms; all the individuals lived in the community. Most of individuals were co-operative during the examination.

In Table 1 all evaluation of all parameters considered for participants with DS are reported with reference values for normal individuals.

BMI, WHER and cholesterol HDL values were higher than the recommended range in the people with DS; on the contrary, blood pressure (systolic and diastolic), heart rate, glycaemia, cholesterol-LDL and triglycerides were within normal levels, although heart rate was skewed towards the lower end of the normal distribution. Comparing males and females in the DS group, the females showed different values from a statistical point of view for cholesterol-total (males showed higher values than females and recommended values, while females were within normal range) and triglycerides values for both males and females were inside normality, although females had lower values in comparison to males. In terms of cholesterol-LDL, no statistical differences existed between males and females but in males, the values were skewed towards the higher end of the normal distribution. In motor performance, people with DS presented abnormal gait patterns as demonstrated by GPS values being higher than reference values. No other statistical differences were found.

The following significant correlations $(p<0.05)$ were found: 
Table 1: Mean and Standard Deviation Values of Clinical, Cardiac and Gait Pattern Parameters for Males and Females of Group with DS. Reference Values were Reported for each Parameter. ${ }^{*}=p<0.05$, Males vs. Females

\begin{tabular}{|c|c|c|c|}
\hline \multirow{2}{*}{ Parameters } & \multicolumn{2}{|c|}{ Group with DS } & \multirow{2}{*}{ Reference values $[26,34,35]$} \\
\hline & Males & Females & \\
\hline Age (years) & $37.30(6.47)$ & $34.53(8.20)$ & --- \\
\hline BMI (Kg/m2) & $27.26(4.37)$ & $28.72(4.31)$ & $<25$ \\
\hline WHER & $0.61(0.07)$ & $0.63(0.08)$ & $<0.49$ \\
\hline Heart rate (beats/minute) & $61.40(11.46)$ & $62.00(10.33)$ & $60-90$ \\
\hline Systolic blood pressure $(\mathrm{mmHg})$ & $103.13(11.09)$ & $102.67(8.21)$ & $<140$ \\
\hline Diastolic blood pressure $(\mathrm{mmHg})$ & $67.38(5.68)$ & $66.33(7.19)$ & $<90$ \\
\hline Glycaemia (mg/dl) & $85.38(7.05)$ & $85.88(13.47)$ & $70-100$ \\
\hline Cholesterol-Total (mg/dl) & $221.75(34.69)^{*}$ & $184.63(26.99)$ & $<200$ \\
\hline Cholesterol-HDL (mg/dl) & $47.13(6.66)$ & $56.63(12.30)$ & $>36$ \\
\hline Cholesterol-LDL (mg/dl) & $131.72(40.23)$ & $98.20(63.27)$ & $50-130$ \\
\hline Triglycerides(mg/dl) & $151.63(59.29)^{*}$ & $79.25(24.32)$ & $15-200$ \\
\hline Gait Profile Score (degrees) & $9.96(2.07)$ & $10.93(4.20)$ & $<6$ \\
\hline
\end{tabular}

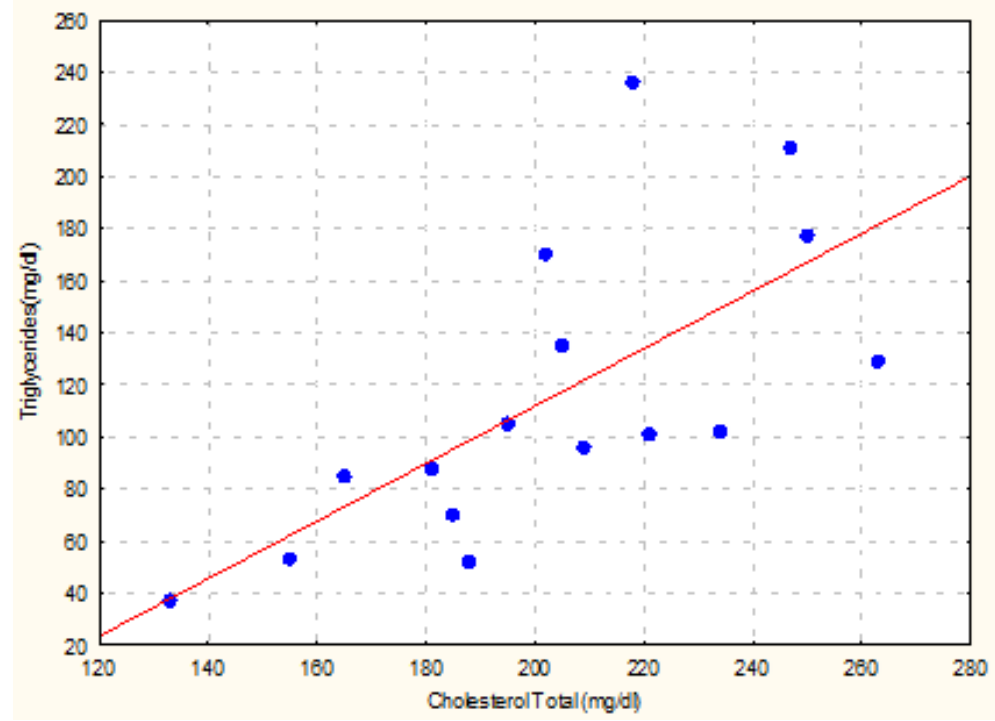

Figure 1: Correlation between Cholesterol Total and Triglycerides values $(r=-0.76, p<0.05)$.

- Age with LDL $(r=0.61)$

- WHER with weight $(r=0.73)$ and BMI $(r=0.85)$

- Systolic with diastolic blood pressure $(r=0.52)$

- Total cholesterol with LDL $(r=0.59)$ and triglycerides $(r=.076)$ (Figure 1)

\section{DISCUSSION}

The health of people with DS has, until recently, been of low priority, although adults with Down syndrome are living longer than previously and individuals surviving into their sixth or seventh decade of life are now encountered [28]. With increased longevity, such individuals are susceptible to different medical and psychiatric disorders, including visual impairment [29], hearing loss [30], thyroid dysfunction [31], dementia [28] and depression [32]. Consequently, there is a need for regular health assessments of adults with DS.

The aim of this research was first to describe the cardiac features in a group of adult persons with DS. Congenital heart defects are well documented in 
literature, but little evidence is reported about acquired cardiac factors. To our knowledge, in fact, only one dated paper [25] assessed adults with DS from this point of view. No other recent studies were conducted on this topic of interest. Then, we aimed to search for a possible correlation between cardiac parameters, presence of obesity and gait alterations.

In the present study, $78 \%$ (29/37 participants) of the examined individuals were found to be overweight or obese (BMI >24). Obesity appears to be particularly associated with people with DS, although other disorders are of a similar magnitude to those found in all people with learning disabilities as a whole. Our results for systolic and diastolic blood pressure were similar to literature [25]. Prasher [25] found the mean systolic reading for the sample population measured was $115 \mathrm{mmHg}$ and a mean diastolic reading of 75 $\mathrm{mmHg}$ with no sex difference. They found a significant positive correlation between increased age and increase in systolic and diastolic blood pressure readings, results not found with our data, probably due to the restricted sample we analysed. We did not find any diastolic readings over $90 \mathrm{mmHg}$ or systolic readings over $150 \mathrm{mmHg}$, findings comparable to Prasher [25].

Heart rates were skewed towards the lower end of control values. The mean resting heart rate for the general population is 72 beats/min (range 60-90). Our findings suggest that adults with DS have lower resting heart rates than controls [25]. Data for glycaemia and triglycerides were close to reference values. Cholesterol-LDL and total cholesterol were higher in males than females, with cholesterol-LDL skewed towards the higher end of the normal distribution and total values out of reference range. No evidence is present in the literature about these parameters; so, comparison with previous data is not possible.

We analysed gait pattern in participants with DS using a summary measure, the GPS, obtained by quantitative analysis of walking (Gait Analysis). Individuals with DS presented abnormal gait patterns, as the value of the GPS was higher than the reference value, confirming reports in the literature [12, 14, 33]. No significant correlations were found between GPS and BMI, WHER and cardiac parameters. The lack of correlations between functional limitations during gait analysis, quantified by GPS, BMI and WHER, correlated with the presence or absence of obesity, showed that obesity did not strong influence the gait pattern in participants with DS. The gait alterations found in individuals with DS seem to be associated more with the specific syndrome and less with the presence/absence of obesity. A recent study quantified the differences during gait between obese individuals with DS and non obese individuals with DS [21]. The results of this study showed that obesity in individuals with DS has an influence on the gait strategy: obese patients with DS showed higher duration of stance phase and abnormalities at ankle joint (reduced ankle dorsiflexion ability in swing phase and limited ankle joint stiffness) [21]. Our results seem to be in contrast with literature. It is important to underline that in the present research the gait pattern was quantified using a summary parameter, the GPS, which is a single index outcome measure that summarises the overall quality of the patient's kinematics [26], including all the lower limb joints. It could be the reason of the absence of significant association between BMI and gait pattern found in the present research.

As demonstrated by this study and from previous research, adults with DS suffer from a high prevalence of physical disorders. From a clinical point of view, significant physical disorders include overweight, obesity and abnormal cholesterol values. As individuals with DS may not be able to communicate pain or discomfort associated with a given illness, ill health may be masked, resulting in missed or misdiagnosis of psychiatric and physical illness. Complications may follow, potentially, proving to be fatal.

In order to ensure, therefore, that appropriate healthcare is provided to adults with DS, health services must be aware of the diagnosis, treatment and management of such medical disorders, occurring in this population. An active ongoing screening programme must be implemented. Individuals themselves, their parents and caregivers need to be educated about the health difficulties and disorders needing to be monitored.

With the improved life expectancy of people with DS, older individuals are more vulnerable to physical, sensory and psychiatric morbidity. The development of a high quality comprehensive health monitoring service is essential to ensure a good quality of life.

This study has several limitations. First, the research was conducted in a small sample across a large age span. These elements surely influence statistical power. In addition, it would be useful to divide the participants in subgroups according for example to the age, as cardiac features vary significantly with age; 
in this research, it was not possible because a limited number of subjects would have been included in each subgroups, further reducing the statistical power. However, to our knowledge no recent studies were conducted on this topic and in particular on the research for a possible correlation between cardiac parameters, obesity and gait alterations. Further researches should be conducted with a larger sample, to have the possibility to create subgroups according to age, and considering control group of age-matched.

\section{COMPETING INTEREST}

All authors haven't any conflicts of interest and any financial interest. All authors attest and affirm that the material within has not been and will not be submitted for publication elsewhere.

\section{REFERENCES}

[1] Hassold T, Abruzzo M, Adkins K, et al. Human aneuploidy: incidence, origin, and etiology. Environmental and Molecular Mutagenesis 1996; 28: 167-75.

https://doi.org/10.1002/(SICI)10982280(1996)28:3<167::AID-EM2>3.0.CO;2-B

[2] O'Nuallain S, Flanagan O, Raffat I, Avalos G, Dineen B. The prevalence of Down syndrome in County Galway. Irish Medical Journal 2007; 100: 329-331.

[3] Canfield MA, Honein MA, Yuskiv N, et al. National estimates and race/ethnic-specific variation of selected birth defects in the United States, 1999-2001. Birth Defects Research Part A: Clinical and Molecular Teratology 2006; 76: 747-756. https://doi.org/10.1002/bdra.20294

[4] Murthy SK, Malhotra AK, Mani S, et al. Incidence of Down syndrome in Dubai, UAE. Medical Principles and Practice 2007; 16: 25-28.

https://doi.org/10.1159/000096136

[5] Morris JK, Wald NJ, Watt HC. Fetal loss in Down syndrome pregnancies. Prenatal Diagnosis 1999; 19: 142-145.

https://doi.org/10.1002/(SICl)10970223(199902)19:2<142::AID-PD486>3.0.CO;2-7

[6] ENCODE Project Consortium. Identification and analysis of functional elements in $1 \%$ of the human genome by the ENCODE pilot project. Nature 2007; 447; 799-816.

[7] Antonarakis SE, Lyle R, Dermitzakis ET, Reymond A, Deutsch S. Chromosome 21 and Down syndrome: from genomics to pathophysiology. Nature Reviews Genetics 2004; 5: 725-738.

https://doi.org/10.1038/nrg1448

[8] Agiovlasitis S, McCubbin JA, Yun J, Mpitsos G, Pavol MJ. Effects of Down syndrome on three-dimensional motion during walking at different speeds. Gait Posture 2009; 30: 345-50.

https://doi.org/10.1016/j.gaitpost.2009.06.003

[9] Caird MS, Wills BPD, Dormans JP. Down syndrome in children: The role of the orthopaedic surgeon. Journal of the American Academy of Orthopaedic Surgeons 2006; 14: 610619.

https://doi.org/10.5435/00124635-200610000-00003

[10] Caselli MA, Cohen-Sobel E, Thompson J, Adler J, Gonzalez L. Biomechanical management of children and adolescents with Down syndrome. Journal of the American Podiatric Medical Association 1991; 81: 119-27.

https://doi.org/10.7547/87507315-81-3-119
[11] Cimolin V, Galli M, Grugni G, et al. Gait patterns in PraderWilli and Down syndrome patients. Journal of Neuro Engineering and Rehabilitation 201; 21, 7: 28.

[12] Cimolin V, Galli M, Grugni G, et al. Postural strategies in Prader-Willi and Down syndrome patients. Research in Developmental Disabilities 2011; 32: 669-73. https://doi.org/10.1016/j.ridd.2010.11.017

[13] Concolino D, Pasquzzi A, Capalbo G, Sinopoli S, Strisciuglio $P$. Early detection of podiatric anomalies in children with Down syndrome. Acta Paediatrica 2006; 95: 17-20.

[14] Galli M, Rigoldi C, Brunner R, Virji-Babul N, Albertini G. Joint stiffness and gait pattern evaluation in children with Down syndrome. Gait Posture 2008; 28: 502-506. https://doi.org/10.1016/j.gaitpost.2008.03.001

[15] Galli M, Cimolin V, Pau M, Costici P, Albertini G. Relationship between flat foot condition and gait pattern alterations in children with Down syndrome. Journal of Intellectual Disability Research 2014; 58: 269-76. https://doi.org/10.1111/jir.12007

[16] Galli M, Cimolin V, Rigoldi C, Pau M, Costici P, Albertini G. The effects of low arched feet on foot rotation during gait in children with Down syndrome. Journal of Intellectual Disability Research 2014; 58: 758-64. https://doi.org/10.1111/jir.12087

[17] Mahan KT, Diamond E, Brown D. Podiatric profile of the Down's syndrome individual. Journal of the American Podiatric Medical Association 1983; 73: 173-9. https://doi.org/10.7547/87507315-73-4-173

[18] Mik G, Gholve PA, Scher DM, Widmann RF, Green DW. Down syndrome: orthopedic issues. Current Opinion in Pediatrics 2008; 20: 30-6. https://doi.org/10.1097/MOP.0b013e3282f35f19

[19] Steingass KJ, Chicoine B, McGuire D, Roizen NJ. Developmental disabilities grown up: Down syndrome. Journal of Developmental \& Behavioral Pediatrics 2011; 32: 548-58.

https://doi.org/10.1097/dbp.0b013e31822182e0

[20] Weijerman ME, de Winter JP. Clinical practice. The care of children with Down syndrome. European Journal of Pediatrics 2010; 169: 1445-52. https://doi.org/10.1007/s00431-010-1253-0

[21] Galli M, Cimolin V, Rigoldi C, Condoluci C, Albertini G. Effects of obesity on gait pattern in young individuals with Down syndrome. International Journal of Rehabilitation Research 2015; 38: 55-60. https://doi.org/10.1097/MRR.0000000000000092

[22] Bean LJ, Allen EG, Tinker SW, et al. Ethnicity, sex, and the incidence of congenital heart defects: a report from the National Down Syndrome Project. Genetics in Medicine 2008; 10: 173-180.

https://doi.org/10.1097/GIM.0b013e3181634867

[23] Morrison RA, McGrath A, Davidson G, Brown JJ, Murray GD, Lever AF. Low blood pressure in Down's syndrome, a link with Alzheimer's disease? Hypertension 1996; 28: 569-575.

[24] Draheim CC, McCubbin JA, Williams DP. Differences in cardiovascular disease risk between nondiabetic adults with mental retardation with and without Down syndrome. American Journal on Mental Retardation 2002; 107: 201-211. https://doi.org/10.1352/08958017(2002)107<0201:DICDRB >2.0.CO;2

[25] Prasher VP. Prevalence of thyroid dysfunction and autoimmunity in adults with Down syndrome. Down's Syndrome Research and Practice 1994; 2: 67-70. https://doi.org/10.3104/reports.32

[26] Baker R, McGinley JL, Schwartz MH, et al. The gait profile score and movement analysis profile. Gait Posture 2009; 30: 265-9.

https://doi.org/10.1016/j.gaitpost.2009.05.020 
[27] Cimolin V, Galli M. Summary measures for clinical gait analysis: a literature review. Gait Posture 2014; 39: 1005-10. https://doi.org/10.1016/i.gaitpost.2014.02.001

[28] Prasher VP, Krishnan VHR. Age of onset and duration of dementia in people with Down syndrome. International Journal of Geriatric Psychiatry 1993; 8: 923-927. https://doi.org/10.1002/gps.930081106

[29] Hestnes A, Sand T, Fostad K. Ocular findings in Down's syndrome. Journal of Mental Deficiency Research 1991; 35: 194-203. https://doi.org/10.1111/j.1365-2788.1991.tb01052.x

[30] Evenhuis HM, van Zanten GA, Brocaar MP, Roerdinkholder WH. Hearing loss in middle-age persons with Down syndrome. American Journal on Mental Retardation 1992; 97: 47-56.

[31] Dinani S, Carpenter S. Down's syndrome and thyroid disorder. Journal of Mental Deficiency Research 1990; 34: 187-93. https://doi.org/10.1111/j.1365-2788.1990.tb01528.x
[32] Burt DB, Loveland KA, Lewis KR. Depression and the onset of dementia in adults with mental retardation. American Journal on Mental Retardation 1992; 96: 502-11.

[33] Galli M, Cimolin V, Rigoldi C, Kleiner A, Condoluci C Albertini G. Use of the Gait Profile Score for the quantification of gait pattern in Down Syndrome. Journal of Developmental and Physical Disabilities 2015; 27: 609-615. https://doi.org/10.1007/s10882-015-9438-0

[34] Expert Panel on Detection, Evaluation, and Treatment of High Blood Cholesterol in Adults. Executive Summary of The Third Report of The National Cholesterol Education Program (NCEP) Expert Panel on Detection, Evaluation, And Treatment of High Blood Cholesterol In Adults (Adult Treatment Panel III). Journal of the American Medical Association, 2001; 285: 2486-97. https://doi.org/10.1001/jama.285.19.2486

[35] Sauer WH. Normal sinus rhythm and sinus arrhythmia. Available from: http: //www.uptodate.com/index.

DOI: https://doi.org/10.6000/2292-2598.2017.05.01.3

(c) 2017 Condoluci et al.; Licensee Lifescience Global.

This is an open access article licensed under the terms of the Creative Commons Attribution Non-Commercial License (http://creativecommons.org/licenses/by-nc/3.0/) which permits unrestricted, non-commercial use, distribution and reproduction in any medium, provided the work is properly cited. 